\title{
Necessary and sufficient conditions for local creation of quantum correlation
}

\author{
Xueyuan $\mathrm{Hu}$ * Heng Fan, D. L. Zhou, and Wu-Ming Liu \\ Beijing National Laboratory for Condensed Matter Physics, \\ Institute of Physics, Chinese Academy of Sciences, Beijing 100190, China
}

(Dated: November 2, 2018)

\begin{abstract}
Quantum correlation can be created by local operation from some initially classical states. We prove that the necessary and sufficient condition for a local trace-preserving channel to create quantum correlation is that it is not a commutativity-preserving channel. This condition is valid for arbitrary finite dimension systems. We also derive the explicit form of commutativity-preserving channels. For a qubit, a commutativity-preserving channel is either a completely decohering channel or a mixing channel. For a three-dimension system (qutrit), a commutativity-preserving channel is either a completely decohering channel or an isotropic channel.

PACS numbers: 03.65.Ud, 03.65.Yz, 03.67.Mn
\end{abstract}

Quantum correlation is the unique phenomenon of quantum physics and believed to be a resource for quantum information processes which can generally surpass the corresponding classical schemes. Many previous studies focus on entanglement, a well-known quantum correlation, since its apparent role in teleportation, superdense coding [1, 2], etc. Recently, measures of the nonclassicalness of correlation, such as quantum discord [3] and quantum deficit [4, 5], began to attract much attention since the discovery that some quantum information schemes can be realized without entanglement but with a positive quantum discord [6, 7]. Much progress has been made to quantify the amount of quantum correlation in different physical systems [8, 9] and to give it intuitive and operational interpretations. It is shown that quantum discord can be operationally interpreted as the minimum information missing from the environment [10]. One-way quantum deficit [11, 12] has been found as the reason for entanglement irreversibility 13] and can be related to quantum entanglement via an interesting scheme [14, 15].

Quantum noise usually plays a destructive role in quantum information process. However, there are situations that local quantum noise can enhance nonlocal quantum properties for some mixed quantum states. For example, local amplitude damping can increase the average teleportation fidelity for a class of entangled states [16 18]. Quantum discord can also be increased or created by local noise [19 21]. An interesting result is that any separable state with positive quantum discord can be produced by local positive operator-valued measure (POVM) on a classical state in a larger Hilbert space 22]. In fact, almost all states in the Hilbert space contains quantum correlation, and an arbitrary small disturbance can drive a classical state into a quantum state with nonzero quantum correlation [23]. Counterintuitively, it has recently been discovered that mixedness is as important as entanglement for quantum correlation. In partic-

*Electronic address: xyhu@iphy.ac.cn ular, some mixed states contain more quantum discord than that of maximally entangled pure state when the dimension of the system is large enough [15]. Thus it is of interest to know how is the effect of mixedness on the quantum correlation of quantum states. The condition for local increase of quantum correlation has been derived for the qubit case [24], and it has been pointed out that this condition is not valid for high-dimension systems.

In this article, we derive a simple necessary and sufficient condition for a local channel to create quantum correlation in some half-classical states, which is valid for arbitrary finite dimension systems. A trace-preserving local channel can create quantum correlation if and only if it is not a commutativity-preserving channel. For qubit case, we show that a commutativity-preserving channel is either a mixing channel or a completely decohering channel. This confirms the result in Ref. [24]. For the qutrit case, quantum correlation can be created by a local channel in some half-classical input states if and only if the channel is neither a completely decohering channel nor an isotropic channel. We also analyze the reason for a local mixing channel to create quantum correlation in qutrit situation and then give a conjecture to extend the result of qutrits to arbitrary finite dimension systems.

The total correlation between two quantum systems is composed of classical and quantum correlations. From this point of view, quantum correlation is defined as the difference between total and classical correlations. Therefore, various measures of quantum correlation defined on one party of a composite system vanish for exact the same class of states, which is called half-classical states. Because classical correlation is defined by the correlation that can be revealed by local measurements, a state $\rho_{A B}$ is half-classical on $B$ if and only if there exist a measurement on $B$ that does not affect the total state. As proved in Ref. 25] a half-classical state on $B$ can be written as

$$
\rho_{A B}=\sum_{i} p_{i} \rho_{A}^{\alpha_{i}} \otimes\left|\alpha_{i}\right\rangle_{B}\left\langle\alpha_{i}\right|
$$

where $\left\{\left|\alpha_{i}\right\rangle_{B}\right\}$ consist an orthogonal basis for the Hilbert space of subsystem $B$, and $\rho_{A}^{\alpha_{i}}$ are corresponding density 
matrices of $A$. The subsystem $A$ can be a single quantum particle or an ensemble of quantum particles. In the following, by quantum correlation, we mean quantum correlation defined on subsystem $B$. The main purpose of this paper is to characterize the channel $\Lambda_{B}$ satisfying

$$
\mathrm{I}_{A} \otimes \Lambda_{B}\left(\rho_{A B}\right) \in \mathcal{D}_{0}, \forall \rho_{A B} \in \mathcal{D}_{0},
$$

where $\mathcal{D}_{0}$ is the set of half-classical states. Before providing the condition, we first introduce a class of quantum channels, which we call commutativity-preserving channels.

Definition 1 (commutativity-preserving channel): a commutativity-preserving channel $\Lambda^{\mathrm{CP}}$ is the channel that can preserve the commutativity of any input density operators, i.e.,

$$
\left[\Lambda^{\mathrm{CP}}(\xi), \Lambda^{\mathrm{CP}}\left(\xi^{\prime}\right)\right]=0
$$

holds for any density operators satisfying $\left[\xi, \xi^{\prime}\right]=0$.

It is worth mentioning an equivalent definition of a commutativity-preserving channel. A channel $\Lambda$ is a commutativity-preserving channel if and only if

$$
[\Lambda(\phi), \Lambda(\psi)]=0
$$

holds for any pure states satisfying $\langle\phi \mid \psi\rangle=0$. The "only if" part is obtained directly by choosing $\xi=|\phi\rangle\langle\phi|$ and $\xi^{\prime}=|\psi\rangle\langle\psi|$. Conversely, if Eq. (4) holds, by writing $\xi$ and $\xi^{\prime}$ on their common eigenbasis, we arrive at Eq. (3).

Now we are ready to prove the first main result of this paper. It holds for arbitrary finite-dimension systems.

Theorem 1: A channel $\Lambda$ acting on subsystem $B$ can create quantum correlation between subsystems $A$ and $B$ for some input half-classical state $\rho_{A B}$ if and only if it is not a commutativity-preserving channel.

Proof: Any separable state can be written as

$$
\xi_{A B}=\sum_{i} p_{i} \xi_{i}^{A} \otimes \xi_{i}^{B}
$$

where $\xi_{i}^{A}$ are linearly independent. We will first prove that $\xi_{A B}$ is a half-classical state if and only if

$$
\left[\xi_{i}^{B}, \xi_{j}^{B}\right]=0, \forall i, j .
$$

For proving the "only if" part, we notice that for any half-classical state, there exist a measurement basis $\Pi_{B}^{\alpha_{i}}$ that does not affect the state. Therefore,

$$
\sum_{i} p_{i} \xi_{i}^{A} \otimes\left(\xi_{i}^{B}-\Pi_{B}^{\alpha_{j}} \xi_{i}^{B} \Pi_{B}^{\alpha_{j}}\right)=0 .
$$

Because $\xi_{i}^{A}$ are linearly independent, $\xi_{i}^{B}$ is diagonal on $\left\{\Pi_{B}^{\alpha_{j}}\right\}$ and thus satisfies Eq. (6). Conversely, if Eq. (6) holds, $\xi_{i}^{B}$ and $\xi_{j}^{B}$ share common eigenvectors for any $i$ and $j$. By choosing these eigenvectors as the basis for von Neumann measurement, the state does not change after the measurement, which means that $\xi_{A B}$ is a halfclassical state. Now consider an arbitrary half-classical state in form of Eqs. (5) and (6) as the input state, the channel $\Lambda$ acting on subsystem $B$ leads the state to $\xi_{A B}^{\prime} \equiv \mathrm{I}_{A} \otimes \Lambda_{B}\left(\xi_{A B}\right)=\sum_{i} p_{i} \xi_{i}^{A} \otimes \Lambda\left(\xi_{i}^{B}\right)$, which is still a half-classical state if and only if

$$
\left[\Lambda\left(\xi_{i}^{B}\right), \Lambda\left(\xi_{j}^{B}\right)\right]=0
$$

for arbitrary choice of $\xi_{i}^{B}$ and $\xi_{j}^{B}$ satisfying Eq. (6). This is just the definition of a commutativity-preserving channel. Therefore, the channel $\Lambda$ can create quantum correlation for some input half-classical states if and only if it is not a commutativity-preserving channel. This completes the proof.

The rest of this paper is devoted to expose the exact form of a commutativity-preserving channel.

Since $[\mathrm{I}, \rho]=0, \forall \rho$, we obtain a necessary condition for a commutativity-preserving channel

$$
[\Lambda(\mathrm{I}), \Lambda(\rho)]=0, \forall \rho .
$$

When $B$ is a qubit, Eq. (9) is also the sufficient condition. The reason is as follows. By using the linearity of $\Lambda$, the left hand side of Eq. (4) can be written as

$$
\begin{aligned}
& \frac{1}{2}[\Lambda(|\phi\rangle\langle\phi|+| \psi\rangle\langle\psi|), \Lambda(|\psi\rangle\langle\psi|-| \phi\rangle\langle\phi|)] \\
= & \frac{1}{2}\left[\Lambda(\mathrm{I}), \Lambda\left(u \sigma^{z} u^{\dagger}\right)\right],
\end{aligned}
$$

where $|\psi\rangle=u|0\rangle$ and $|\phi\rangle=u|1\rangle$. Since any qubit state $\rho$ can be decomposed as $\rho=\left(\mathrm{I}+n_{x} \sigma_{x}+n_{y} \sigma_{y}+n_{z} \sigma_{z}\right) / 2$, Eq. (4) is equivalent to Eq. (91). From this observation, we can see that a qubit channel $\Lambda$ is commutativitypreserving if and only if it is one of the following two cases:

Case 1: $\Lambda(\mathrm{I})=\mathrm{I}$, which means that $\Lambda$ is a unital channel. Here we define a mixing channel $\Lambda^{\mathrm{M}}$ as

$$
S\left(\Lambda^{\mathrm{M}}\left(\rho_{S}\right)\right) \geq S\left(\rho_{S}\right), \forall \rho_{S},
$$

where $S(\rho) \equiv-\operatorname{Tr}\left(\rho \log _{2} \rho\right)$ is the von Neumann entropy. It is worth mentioning that when a channel is a mixing channel, its extension to larger systems $\mathrm{I}_{A} \otimes \Lambda_{S}^{\mathrm{M}}$ is still a mixing channel. As proved in Ref. [26], a mixing channel is equivalent to a unital channel.

Case 2: $\Lambda(\mathrm{I}) \neq \mathrm{I}$. Then the diagonal basis of $\Lambda(\mathrm{I})$ is specified. According to Eq. (8), the two matrices $\Lambda(\rho)$ and $\Lambda(\mathrm{I})$ share common eigenvectors. In other words, the channel $\Lambda$ takes any input state $\rho$ to a diagonal form on the eigenbasis of $\Lambda(\mathrm{I})$, and is thus a completely decohering channel.

Therefore, when $B$ is a qubit, a commutativitypreserving channel is either a mixing channel or a completely decohering channel. This confirms the result in Ref. 24].

In the following, we will move on to study the exact form of a commutativity-preserving channel for highdimension cases.

Definition 2 (isotropic channel) An isotropic channel is of the form

$$
\Lambda^{\text {iso }}(\rho)=p \Gamma(\rho)+(1-p) \frac{\mathrm{I}}{d},
$$


where $\Gamma$ is any linear channel that preserves the eigenvalues of $\rho$. According to Ref. [27], $\Gamma$ is either a unitary operation or unitarily equivalent to transpose. Parameter $p$ is chosen to make sure that $\Lambda$ is a completely positive channel. In particular, $-1 /(d-1) \leq p \leq 1$ when $\Gamma$ is a unitary operation, and $-1 /(d-1) \leq p \leq 1 /(d+1)$ when $\Gamma$ is unitarily equivalent to transpose.

Theorem 2: Consider the half-classical input state in Eq. (11) with $B$ a qutrit, a channel $\Lambda$ can not create quantum correlation in any half-classical input state if and only if $\Lambda$ is either a completely dechering channel or an isotropic channel.

Proof: Writing the eigen-decomposition of $\Lambda(\mathrm{I})$ as

$$
\Lambda(\mathrm{I})=\sum_{i=1}^{N} \lambda_{i} \mathrm{I}_{r_{i}} .
$$

Here $\sum_{i=1}^{N} r_{i}=\sum_{i=1}^{N} r_{i} \lambda_{i}=3, \lambda_{i} \geq 0, r_{i}$ are positive integers, and $\mathrm{I}_{r_{i}}$ are identities of the $r_{i}$-dimension subspace $\mathcal{V}_{r_{i}}$. From Eq. (9) we have

$$
\Lambda(\rho)=\sum_{i=1}^{N} q_{i} \xi_{r_{i}}^{\rho}, \forall \rho .
$$

where $\xi_{r_{i}}^{\rho}$ is a density operator on $\mathcal{V}_{r_{i}}$.

Clearly, when the eigenvectors of $\Lambda(\mathrm{I})$ are nondegenerate, i.e., $N=3$ and Eq. (13) becomes $\Lambda(\mathrm{I})=\sum_{i=1}^{3} \lambda_{i} \Pi_{i}$, the channel $\Lambda$ is a completely decohering channel, since it takes any input state $\rho$ to a diagonal form on basis $\left\{\Pi_{i}\right\}$. When two or three eigenvectors of $\Lambda(\mathrm{I})$ are degenerate, we study the eigendecomposition of $\Lambda(\phi)$ for a pure input state $|\phi\rangle$

$$
\Lambda(\phi)=\sum_{i=1}^{N^{\phi}} \lambda_{i}^{\phi} \mathrm{I}_{r_{i}(\phi)},
$$

where $N^{\phi} \geq N$ and $\mathcal{V}_{r_{i}(\phi)} \subseteq \mathcal{V}_{r_{j}}$. When none of $\Lambda(\phi)$ breaks the degeneracy of eigenvectors of $\Lambda(\mathrm{I})$, i.e., $N^{\phi}=$ $N$ and $\mathcal{V}_{r_{i}(\phi)}=\mathcal{V}_{r_{i}}$, the channel is also a completely decohering channel. Now we focus on the case that some $\Lambda(\phi)$ can break the degeneracy of eigenvectors of $\Lambda(\mathrm{I})$, i.e., $N^{\phi}>N$ and $\mathcal{V}_{r_{i}(\phi)} \subset \mathcal{V}_{r_{j}}$ for some $i$. Let $\left\{\left|\phi_{k}\right\rangle\right\}_{k=0}^{2}$ be a basis of the three-dimension Hilbert space and $\left|\phi_{0}\right\rangle$ be the pure input state whose corresponding output state $\Lambda\left(\phi_{0}\right)$ has the most different eigenvalues. It means that $N^{\phi_{0}} \geq N^{\phi}, \forall \phi$.

Case 1: For any state $\left|\phi_{0}^{\perp}\right\rangle=c_{1}\left|\phi_{1}\right\rangle+c_{2}\left|\phi_{2}\right\rangle$ which is orthogonal to $\left|\phi_{0}\right\rangle$, we have $N^{\phi_{0}^{\perp}}=N^{\phi_{0}}$ and $\mathcal{V}_{r_{i}\left(\phi_{0}^{\perp}\right)}=$ $\mathcal{V}_{r_{i}\left(\phi_{0}\right)}$. Then for arbitrary input state $\varphi=\sum_{i=0}^{2} c_{i}\left|\phi_{i}\right\rangle$, we have

$$
\left[\Lambda(\varphi), \Lambda\left(c_{2}^{*}\left|\phi_{1}\right\rangle-c_{1}^{*}\left|\phi_{2}\right\rangle\right)\right]=0 .
$$

Therefore, $\Lambda(\varphi)$ is diagonal on the same basis as $\Lambda\left(\phi_{0}\right)$.

Case 2: There exist a pure state, say $\left|\phi_{2}\right\rangle$, whose corresponding output state $\Lambda\left(\phi_{2}\right)$ does not break as much degeneracy as $\Lambda\left(\phi_{0}\right)$, i.e., $N^{\phi_{2}}<N^{\phi_{0}}$. We will first prove that for any pure state $\left|\varphi_{01}\right\rangle=\left|\phi_{0}\right\rangle-\beta_{0}\left|\phi_{1}\right\rangle$ in 2-dimension subspace $\mathcal{W}_{2}^{\phi_{2}}$, the output state is diagonal on the same basis as $\Lambda\left(\phi_{0}\right)$, say $\left\{\Pi_{i}\right\}$. We introduce $|\varphi(\beta)\rangle=\left|\varphi_{01}\right\rangle+\beta\left|\phi_{2}\right\rangle$ and $\left|\varphi_{02}(\beta)\right\rangle=\beta^{*}\left|\phi_{0}\right\rangle-\left|\phi_{2}\right\rangle$. Notice that $\left\langle\varphi_{02}(\beta) \mid \varphi(\beta)\right\rangle=0$, we have

$$
\left[\Lambda\left(\varphi_{02}(\beta)\right), \Lambda(\varphi(\beta))\right]=0 .
$$

Because $\sum_{k=0}^{2}\left|\phi_{k}\right\rangle\left\langle\phi_{k}\right|=\mathrm{I}$, we have $N^{\phi_{1}}=N^{\phi_{0}}$ and $\mathcal{V}_{r_{i}\left(\phi_{1}\right)}=\mathcal{V}_{r_{i}\left(\phi_{0}\right)}$. Therefore, $\Lambda\left(\varphi_{02}(\beta)\right)$ is diagonal on $\left\{\Pi_{i}\right\}$ by noticing that $\left[\Lambda\left(\varphi_{02}(\beta)\right), \Lambda\left(\phi_{1}\right)\right]=0$. Since the channel cannot increase the distance between states, $\Lambda\left(\varphi_{02}(\beta)\right)$ breaks the same degeneracy as $\Lambda\left(\phi_{0}\right)$ for sufficiently large $|\beta|$. From Eq. (17), we have $\Lambda(\varphi(\beta))$ and $\Lambda(\varphi(-\beta))$ are diagonal on $\left\{\Pi_{i}\right\}$. Therefore, $\Lambda\left(\varphi_{01}\right)=\Lambda(\varphi(\beta))+\Lambda(\varphi(-\beta))-|\beta|^{2} \Lambda\left(\phi_{2}\right)$ is also diagonal on $\left\{\Pi_{i}\right\}$. Further, we will show that $\Lambda(\varphi(\beta))$ is diagonal on $\left\{\Pi_{i}\right\}$ for arbitrary $\beta$. From Eq. (17), this is obvious when $\Lambda\left(\varphi_{02}(\beta)\right)$ is nondegenerate. For the case where $\Lambda\left(\varphi_{02}(\beta)\right)$ is degenerate, $\Lambda\left(\varphi_{02}(-\beta)\right)=$ $|\beta|^{2} \Lambda\left(\phi_{0}\right)+\Lambda\left(\phi_{2}\right)-\Lambda\left(\varphi_{02}(\beta)\right)$ is nondegenerate and consequently, $\Lambda(\varphi(-\beta))$ is diagonal on $\left\{\Pi_{i}\right\}$. Therefore, $\Lambda(\varphi(\beta))=\Lambda\left(\varphi_{01}\right)+|\beta|^{2} \Lambda\left(\phi_{2}\right)-\Lambda(\varphi(-\beta))$ is diagonal on $\left\{\Pi_{i}\right\} . \Lambda$ is a completely decohering channel.

Case 3: now we are only left with the case that $N^{\phi_{0}^{\perp}}=$ $N^{\phi_{0}}$ but $\mathrm{I}_{r_{i}\left(\phi_{0}^{\perp}\right)} \neq \mathrm{I}_{r_{i}\left(\phi_{0}\right)}$, which can happen only when $\Lambda(\mathrm{I})=\mathrm{I}$ and $N^{\phi_{k}}=2$. Therefore, we have

$$
\Lambda\left(\phi_{k}\right)=p \Pi\left(\phi_{k}\right)+(1-p) \frac{\mathrm{I}}{3},
$$

where $\Pi\left(\phi_{k}\right)$ is a basis determined by $\left|\phi_{k}\right\rangle$. Notices that $p$ is independent of $\left|\phi_{k}\right\rangle$ because of the linearity of $\Lambda$. Consequently, for any input state $\rho=\sum_{i} p_{i}\left|\alpha_{i}\right\rangle\left\langle\alpha_{i}\right|$, we have $\Lambda(\rho)=p \sum_{i} p_{i} \Pi\left(\alpha_{i}\right)+(1-p) \mathrm{I} / 3$. It means that channel $\Lambda$ is an isotropic channel.

Combining the three cases together, we conclude that for a qutrit, a commutativity-preserving channel is either a completely decohering channel or an isotropic channel.

Since depolarizing channel is a subset of mixing channel, there exist mixing channels that are able to locally create quantum correlation. Therefore, mixedness can contribute to creation of quantum correlations. Here we give an example to look more closely at why a mixing channel can create quantum correlation in states with high dimensions. Consider the following mixing channel $\Lambda(\cdot)=\sum_{i} \mathrm{E}^{(i)}(\cdot) \mathrm{E}^{(i) \dagger}$, where the Kraus operators are

$$
\begin{aligned}
\mathrm{E}^{(0)} & =|2\rangle\langle 2|, \\
\mathrm{E}^{(i)} & =e_{i} u_{2}^{(i)}(|0\rangle\langle 0|+| 1\rangle\langle 1|), i=1,2, \cdots .
\end{aligned}
$$

Here $u_{2}^{(i)}$ are rank-2 unitary operators on basis $\{|0\rangle,|1\rangle\}$. This channel can create quantum correlation in the state $\rho=\tilde{\rho}_{A}^{\phi} \otimes|\phi\rangle_{B}\left\langle\phi\left|+\tilde{\rho}_{A}^{\psi} \otimes\right| \psi\right\rangle_{B}\langle\psi|$ if and only if Eq. (44) is violated. Writing the two orthogonal states as $|\phi\rangle=\sum_{i=0}^{2} a_{i}|i\rangle$ and $|\psi\rangle=\sum_{i=0}^{2} b_{i}|i\rangle\left(\sum_{i=0}^{2} a_{i} b_{i}^{*}=0\right)$, 
we obtain the left hand side of Eq. (4)

$$
\left[\sum_{i} e_{i}^{2} u_{2}^{(i)}\left|\phi_{2}\right\rangle\left\langle\phi_{2}\left|u_{2}^{(i) \dagger}, \sum_{i} e_{i}^{2} u_{2}^{(i)}\right| \psi_{2}\right\rangle\left\langle\psi_{2}\right| u_{2}^{(i) \dagger}\right]
$$

where $\left|\phi_{2}\right\rangle=a_{0}|0\rangle+a_{1}|0\rangle$ and $\left|\psi_{2}\right\rangle=b_{0}|0\rangle+b_{1}|0\rangle$ are reduced states on Hilbert space of dimension 2. Therefore, Eq. (44) is violated if and only if $\left\langle\phi_{2} \mid \psi_{2}\right\rangle \neq 0,1$. Two high-dimension orthogonal states may become unorthogonal when reduced to Hilbert space of dimension two. This is just the reason for creating quantum correlation using a local mixing channel. Isotropic channels act on all of the states in Hilbert spaces equivalently, so they are likely the only subset of mixing channels which belongs to the class of commutativity-preserving channels. This observation leads to the following conjecture.

Conjecture: Consider the half-classical input state in Eq. (11) where $B$ is a $d$-dimension quantum system (qudit) with $d \geq 3$, a channel $\Lambda$ can not create quantum correlation in any half-classical input state if and only if $\Lambda$ is either a completely dechering channel or an isotropic channel.

We further prove that mixing channel can not increase the teleportation fidelity of any two-qudit state. The average teleportation fidelity $f$ is related to the maximum singlet fraction (MSF) [28] $F=\max _{\Phi}\langle\Phi|\rho| \Phi\rangle$ as $f=$ $(d F+1) /(d+1)$. After the action of mixing channel on $B$, the MSF becomes

$$
F^{\prime}=\operatorname{Tr}(\rho \Xi)
$$

where $\Xi=\sum_{i} \mathrm{I} \otimes \mathrm{E}^{(i) \dagger}|\Phi\rangle\langle\Phi| \mathrm{I} \otimes \mathrm{E}^{(i)}$. Notice that for a mixing channel $\Lambda(\cdot)=\sum_{i} \mathrm{E}^{(i)}(\cdot) \mathrm{E}^{(i) \dagger}$, its conjecture $\Lambda^{*}(\cdot)=\sum_{i} \mathrm{E}^{(i) \dagger}(\cdot) \mathrm{E}^{(i)}$ is also a mixing channel. Therefore, $\Xi_{A}=\Xi_{B}=\mathrm{I} / 2$, so $\Xi$ can be decomposed as a mixture of maximally entangled pure states $\Xi=$ $\sum_{i} p_{i}\left|\Phi_{i}\right\rangle\left\langle\Phi_{i}\right|$. Then we have $F^{\prime}=\sum_{i} p_{i}\left\langle\Phi_{i}|\rho| \Phi_{i}\right\rangle \leq F$. Therefore, average teleportation fidelity can never be increased by mixing channel. This result suggests that quantum correlation created by mixing channel may not be a useful resource for quantum information tasks.

In summary, we have proved that the necessary and sufficient condition for a local operation to create quantum correlation in some half-classical state is that it is not a commutativity-preserving channel. When the subsystem $B$ affected by the local channel is a qubit, a commutativity-preserving channel is either a mixing channel or a completely decohering channel. This result confirms the results in Ref. [24]. When $B$ is a qutrit, we have proved that a commutativity-preserving channel is either an isotropic channel or a completely decohering channel. This result is likely to be extended to arbitrary finite dimension situation.

$\mathrm{Hu}$ thanks Sixia $\mathrm{Yu}$ and Chengjie Zhang for helpful discussions. This work is supported NSFC under grants Nos. 10934010, 60978019, the NKBRSFC under grants Nos. 2009CB930701, 2010CB922904, 2011CB921502, 2012CB821300, NSFC-RGC under grants Nos. 11061160490, 1386-N-HKU748/10, and CNSF under grants Nos. 10975181 and 11175247.
[1] C. H. Bennett, G. Brassard, C. Crépeau, R. Jozsa, A. Peres, and W. K. Wootters, Phys. Rev. Lett. 70, 1895 (1993).

[2] P. Hausladen, R. Jozsa, B. Schumacher, M. Westmoreland, and W. K. Wootters, Phys. Rev. A 54, 1869 (1996).

[3] H. Ollivier and W. H. Zurek, Phys. Rev. Lett. 88, 017901 (2001).

[4] J. Oppenheim, M. Horodecki, P. Horodecki, and R. Horodecki, Phys. Rev. Lett. 89, 180402 (2002).

[5] M. Horodecki, P. Horodecki, R. Horodecki, J. Oppenheim, A. Sen(De), U. Sen, and B. Synak-Radtke, Phys. Rev. A 71, 062307 (2005).

[6] A. Datta, A. Shaji, and C. M. Caves, Phys. Rev. Lett. 100, 050502 (2008).

[7] L. Roa, J. C. Retamal, and M. Alid-Vaccarezza, Phys. Rev. Lett. 107, 080401 (2011).

[8] S. Luo, Phys. Rev. A 77, 042303 (2008).

[9] M. Ali, A. R. P. Rau, and G. Alber, Phys. Rev. A 81, 042105 (2010).

[10] P. J. Coles, arXiv: 1110.1664v1 (2011).

[11] K. Maruyama, F. Nori, and V. Vedral, Rev. Mod. Phys. 81, 1 (2009).

[12] W. H. Zurek, Phys. Rev. A 67, 012320 (2003).

[13] M. F. Cornelio, M. C. de Oliveira, and F. F. Fanchini, Phys. Rev. Lett. 107, 020502 (2011).

[14] A. Streltsov, H. Kampermann, and D. Bruß, Phys. Rev. Lett. 106, 160401 (2011).
[15] M. Piani, S. Gharibian, G. Adesso, J. Calsamiglia, P. Horodecki, and A. Winter, Phys. Rev. Lett. 106, 220403 (2011).

[16] P. Badzia̧g, M. Horodecki, P. Horodecki, and R. Horodecki, Phys. Rev. A 62, 012311 (2000).

[17] Y. Yeo, Phys. Rev. A 78, 022334 (2008).

[18] X. Hu, Y. Gu, Q. Gong, and G. Guo, Phys. Rev. A 81, $054302(2010)$

[19] X. Hu, Y. Gu, Q. Gong, and G. Guo, Phys. Rev. A 84, 022113 (2011).

[20] F. Ciccarello and V. Giovannetti, Phys. Rev. A 85, 010102 (2012).

[21] F. Ciccarello and V. Giovannetti, Phys. Rev. A 85, 022108 (2012).

[22] N. Li and S. Luo, Phys. Rev. A 78, 024303 (2008).

[23] A. Ferraro, L. Aolita, D. Cavalcanti, F. M. Cucchietti, and A. Acín, Phys. Rev. A 81, 052318 (2010).

[24] A. Streltsov, H. Kampermann, and D. Bruß, Phys. Rev. Lett. 107, 170502 (2011).

[25] A. Datta, arXiv:0807.4490v1 (2008).

[26] A. Wehrl, Rev. Mod. Phys. 50, 221 (1978).

[27] M. Marcus and B. N. Moyls, Canad. J. Math. 11, 61 (1959).

[28] M. Horodecki, P. Horodecki, and R. Horodecki, Phys. Rev. A 60, 1888 (1999). 
